\title{
Role of radiologic imaging in genetic and acquired neuromuscular disorders
}

\author{
Paolo Ortolan, Riccardo Zanato, Alessandro Coran, Valeria Beltrame, Roberto Stramare
}

Radiology Unit, Department of Medicine, University of Padova, Italy

\begin{abstract}
Great technologic and clinical progress have been made in the last two decades in identifying genetic defects of several neuromuscular diseases, as Spinal Muscular Atrophy, genetic muscular dystrophies and other genetic myopathies. The diagnosis is usually challenging, due to great variability in genetic abnormalities and clinical phenotypes and the poor specificity of complementary analyses, i.e., serum creatine kinase (CK) and electrophysiology. Muscle biopsy represents the gold standard for the diagnosis of genetic neuromuscular diseases, but clinical imaging of muscle tissue is an important diagnostic tool to identify and quantifyies muscle damage. Radiologic imaging is, indeed, increasingly used as a diagnostic tool to describe patterns and the extent of muscle involvement, thanks to modern techniques that enable to definethe definition of degrees of muscle atrophy and changes in connective tissue. They usually grade the severity of the disease process with greater accuracy than clinical scores. Clinical imaging is more than complementary to perform muscle biopsy, especially as ultrasound scans are often mandatory to identify the muscle to be biopsied. We will here detail and provideWe will herein provide detailed examples of the radiologic methods that can be used in genetic and acquired neuromuscular disorders, stressing pros and cons.
\end{abstract}

Key Words: Muscle Imaging, MRI, CT, genetic muscle disorders, myopathies, dystrophies

Eur J Transl Myol - Basic Appl Myol 2015; 25 (2): 121-127

Great technological and clinical progresses have been made in the last two decades in identifying genetic defects of several neuromuscular diseases, as Spinal Muscular Atrophy, genetic muscle dystrophies and other genetic myopathies. However, the diagnosis is usually challenging, due to great variability in genetic abnormalities and clinical phenotypes, the complexity of the molecular genetic approaches and the poor specificity of complementary analyses, i.e. blood CK, electrophysiology, and others. ${ }^{1}$

Although muscle biopsy represents the gold standard for the diagnosis of genetic neuromuscular diseases, clinical imaging of muscle tissue is an important diagnostic tool for the identification and quantification of muscle changes. ${ }^{2}$ Radiologic imaging is, indeed, increasingly used as a diagnostic tool to describe patterns and the extent of muscle involvement, thanks to modern techniques that enable the definition of degrees of muscle atrophy and changes in connective tissue. They usually grade the severity of the disease process with greater accuracy than clinical scores. Further, clinical imaging is more than complementary to perform muscle biopsy, especially as ultrasound scans are often mandatory to identify the muscle to be biopsied..$^{3-7}$ We will herein provide detailed examples of the several radiologic methods that can be used, stressing for each one their respective pros and cons.

\section{Computer Tomography}

Computer Tomography (CT) has been widely used to assess the presence and extent of changes in skeletal muscle of patients with congenital neuromuscular diseases. $^{8,9}$ In particular, the use of CT has made it possible to detect the selective involvement of muscle groups; moreover, CT has been useful in establishing disease progression and identifying asymptomatic patients. ${ }^{10}$ The myopathic patients usually undergo gradual loss of muscle strength, which doesn't affect every muscle group to the same degree. Selective involvement of specific muscle groups and savings of others has been confirmed by different studies using CT. ${ }^{8,9}$ Fatty replacement of skeletal muscle tissue is seen as an area with a clear decrease in density, ${ }^{2}$ therefore affected muscles are less identifiable as a consequence of the loss of contrast with the subcutaneous adipose tissue. ${ }^{4}$ A CT scan is usually more accessible than magnetic resonance (RM), and it allows for the quick and correct evaluation of muscle 


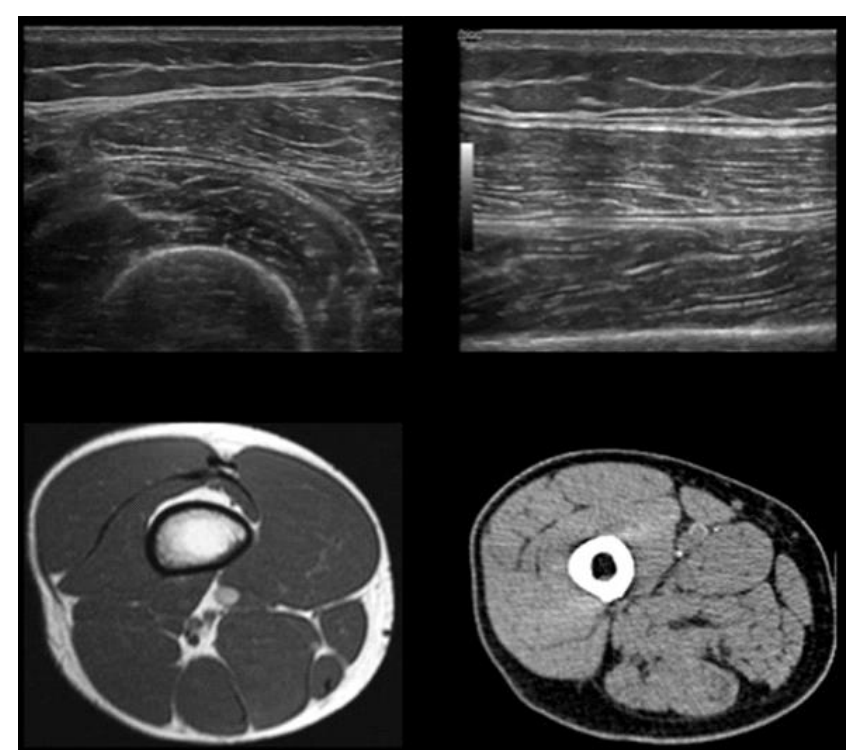

Fig 1. Normal appearance of the tight on ultrasound, computed tomography and magnetic resonance scans at the level of the middle third: in the ultrasound images (axial and longitudinal scans), the normal muscles are characterized by heterogeneous ecostructure and are surrounded by slightly hyperechoic fat; in the CT image, the normal muscle is slightly iperdense (10 to $40 \mathrm{HU})$, while fat tissue is ipodense (-100 to $-50 \mathrm{HU})$; in the MR image (T1-weighted), the normal muscular appearance is characterized by a homogeneous isointense ("grayish") signal intensity. The subcutaneous soft tissues, the intermuscular septa, the intramuscular aponeurosis, and the cancellous bone as well, have a brighter hyperintense signal intensity due to the presence of fat; the femoral cortex is hypointense, the typical signal intensity of cortical bone.

changes, especially fatty degeneration, by assessing muscle density and morphology. Examination is not operator-dependent and allows for the evaluation of the deepest muscles. However, CT scanning needs high doses of ionizing radiation, and has therefore it has been almost entirely replaced by ultrasound (US) and magnetic resonance imaging (MRI). Another drawback are the poor contrasts of the soft tissues, which makes identification of inflammatory changes difficult or impossible; as rule of thumb, muscle edema appears before the muscle degeneration.,11 Furthermore, though TC provides objective information that is easy to be duplicated by different observers and different apparatuses, some muscle artifacts may appear in muscle adjacent to cortical bone tissue. ${ }^{5}$

\section{Ultrasound}

Ultrasound is a well-known validated imaging technique for the evaluation of suspected muscle

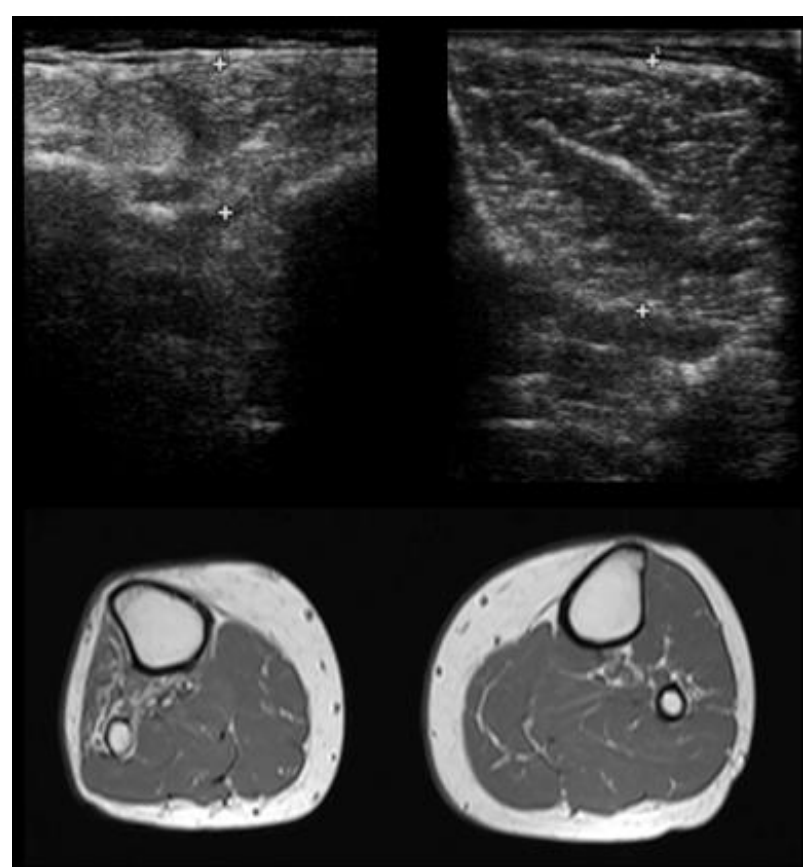

Fig 2. Fatty replacement of the anterolateral compartment muscles of the leg following common fibular nerve injury: ultrasound and magnetic resonance axial images at the level of the middle third of the tibialis anterior belly. The right anterolateral compartment muscles are denervated, so, compared to the contralateral ones, these muscles are atrophic with increase of intramuscular fat: in the ultrasound images, there is a marked thinning of the tibialis anterior muscle with increased echogenicity and loss of physiological heterogeneity of the ecostructure; in the T1weighted MR image, these muscles are brighter.

diseases. It is especially useful with children, due to the lack of ionizing radiations. ${ }^{11,12}$ It is typically used to identify muscle thickness and to evaluate echogenicity changes, and can identify both atrophic changes and fatty degeneration, which is a typical feature of neuromuscular dystrophic diseases, like Duchenne Muscle Dystrophy. Its applications range from initial assessment, ultrasound-guided biopsy and follow-up of the patients (Figs. 1 and 2). Guiding biopsy with US, allows for the targeting the more affected muscles. ${ }^{12-15}$ On top of being low-cost and largely available, ultrasound scans have good resolutions, up to $1 \mathrm{~mm}$, which allows for the identification of tertiary muscular bundles. ${ }^{16}$ US also enables dynamic analysis of muscle contraction by short videos to detect muscle contraction and/or pathologic fasciculations. ${ }^{16}$

Unfortunately, it also has severe disadvantages: its application is usually limited to superficial muscle 


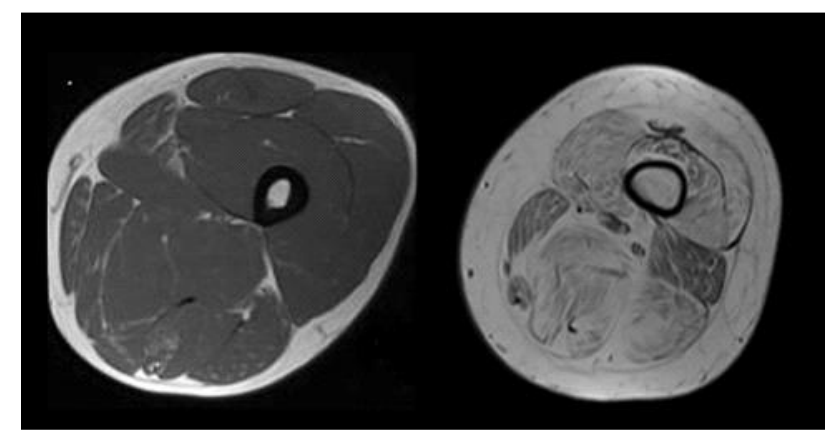

Fig.3 Fatty degeneration: axial T1-weighted images of the thigh showing, on the left, the normal muscular appearance on MR, characterized by a homogeneous grayish ("isointense") signal intensity. On the right, there is a severe and diffuse muscular fatty degeneration: these muscles are hyperintense compared to the previous ones because of the increase of intramuscular fat.

groups, since it is not sufficiently effective in deep muscles evaluation. ${ }^{17}$ Moreover, the inter-observer and intra-observer agreements are really low, given that the muscle morphology and echogenicity are heavily dependent from the manual dexterity of the operator, thus, it is an operator-dependent examination. ${ }^{8}$

\section{Magnetic Resonance}

Due to drawbacks of CT and US, MR is increasingly used in the evaluation of patients with genetic neuromuscular diseases. The technique provides excellent soft tissue characterization, so it perfectly evaluates shape, volume and morphological features of normal skeletal muscle. ${ }^{8}$ Specifically, this technique easily allows for determination of fatty degeneration in late-stage muscle dystrophy, aside from recognition of decreases in muscle volume. Thanks to this optimal soft tissue characterization, MRI easily distinguishes the different muscles in the same muscle group, which is important in the identification of a specific pattern of involvement. ${ }^{8}$ Using qualitative grading, MRI can assess the degree of fat replacement of muscles and thus follow disease progression. Fatty degeneration is usually evaluated in the T1-weighted images, where it appears as area of signal hyperintensity (Fig 3). 2,18

T2-weighted images with fat signal suppression and STIR sequences identify muscle edema, which appear as a diffuse signal hyperintensity of the involved muscles (Fig. 4). $\left.{ }^{2,5,19}\right]$. This is useful in detecting early signs of neuromuscular diseases, because inflammatory changes are often the first manifestation of the muscle disease, much earlier than fat and scar replacement of the muscle tissue. ${ }^{20}$ Recent studies suggest that the use of intravenous administration of contrast medium would provide better details regarding the detection and extent of muscle involvement, although this leads to a substatial increase of scan time. ${ }^{20}$ MRI has higher sensitivity in detecting muscle dystrophic disorders and

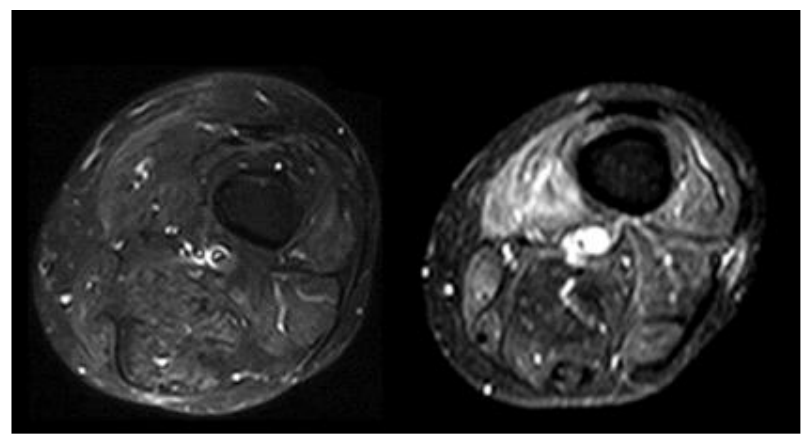

Fig 4. Intramuscular edema: axial STIR images of the thigh showing, on the left, normal muscle signal intensity. In this sequence, the signal coming from the fat is suppressed, so the fat-containing structures are hypointense; on the right, the muscles are hyperintense, particularly the vastus medialis muscle due to the increase of water typical of inflammatory changes.

a good inter-observer and intra-observer agreements; ${ }^{2}$ therefore, MRI is preferred to CT and US in the evaluation of genetic dystrophic neuromuscular disorders, ${ }^{6}$ and also because MRI doesn't expose the patients to ionizing radiation and, thus, can be safely used with young patients. In these cases, RM protocol include Turbo-spin-echo T1-weigthed, in T2-weigthed and in T2 with fat suppression and, eventually STIR (short-tau inversion recovery) sequences. ${ }^{21} \mathrm{MR}$ images are usually acquired in the axial plane with slice thickness of $4 \mathrm{~mm} .{ }^{17}$ When recommended, it is also possible to obtain images according to other anatomical planes (coronal, sagittal) ${ }^{2}$ multiplanar scans, of course, provide more anatomical details. ${ }^{8}$ The typical MR protocol applied in this type of study encompasses T1-weighted scans of the pelvic girdle and lower limbs; there is still little experience in describing neuromuscular diseases by analyzing scans of to chest and upper limbs or by whole-body MRI. ${ }^{22}$ Whole-body MRI can be useful in the assessment of Congenital and Metabolic Myopathies, which typically show muscular involvement of regions other than limbs, without using great doses of ionizing radiation ${ }^{17}$ Myopathic patients usually undergo a partial and gradual loss of muscle strength, but this loss doesn't affect all muscle groups with the same degree of involvement. Selective involvement of certain muscles and and saving of others was confirmed by studies using imaging techniques, in particular $\mathrm{CT}$ and $\mathrm{MR},{ }^{2,4}$ For this reason, it is important to evaluate not only the different muscular compartments, but every single muscle in them, in order to detect specific patterns that can narrow the differential diagnosis and help the search for specific genetic defects, ${ }^{2,22,23}$ for example, some authors have demonstrated specific patterns of involvement in the Central Cores Myopathies, dysferlinopathy (LGMD-2B), hyaline body myopathy (HBM), tubular aggregate myopathy (TAM) and 


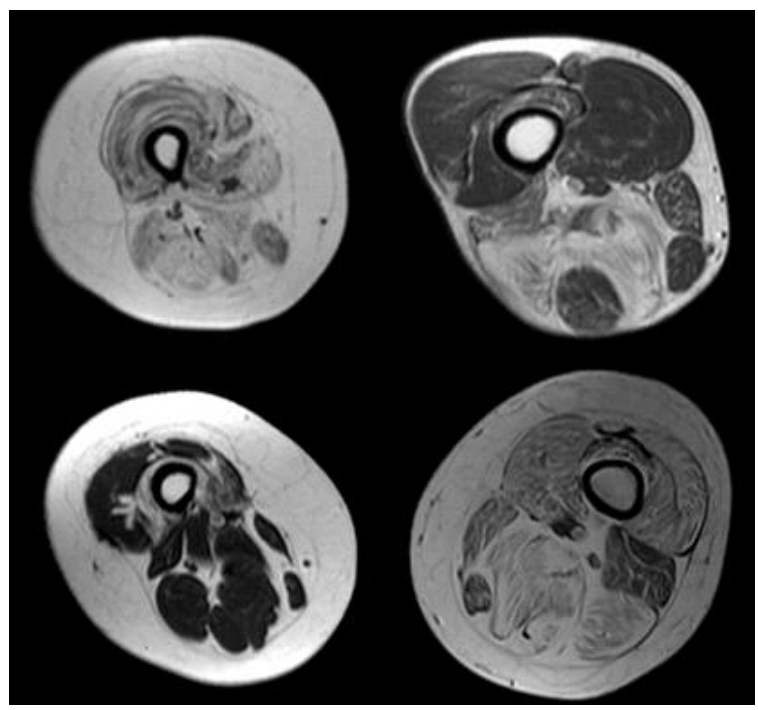

Fig 5. In clockwise order, complete fatty replacement of thigh muscles in limb-girdle muscular dystrophy type B patient (LGMD); "Horseshoelike" muscle fatty replacement in medial and posterior compartments of the thigh in patient with hyaline body myopathy (HBM); crescentshaped fatty degeneration in anterior compartment of the thigh (in particular, vastus intermedius muscle) in myotonic dystrophies type 1 (MD-1); typical degeneration of long and short heads of the biceps femoris in tubular aggregates myopathy (TAM).

myotonic dystrophy (MD) (Fig. 5) ${ }^{21,23}$ while others claim that, by MRI, specific patterns of involvement are powerful predictors in congenital myopathies due to ryanodine receptor type 1 gene mutations. ${ }^{24}$ Due to the lack of ionizing radiation, ${ }^{11} \mathrm{MRI}$ is preferred to CT in young patients and in the assessment of asymptomatic patients with relatives affected by genetic disorders.

There are experimental techniques, both applied to CT and MR, that can quantify the degree of muscle degeneration, evaluate the relationship between muscle fibers and fibrous tissue or measure the amount of intramuscular fat tissue. ${ }^{25-28}$ These techniques can be useful in the evaluation of therapies, but use experimental software and/or MRI sequences that are not easily available and difficult to apply to the clinical practice.

A purpose-developed false-color method of muscle imaging was designed and implemented to analyze macroscopic and microscopic structural changes of human skeletal muscle based on processing techniques of medical CT scans, to follow-up in patients suffering with complete permanent denervation of leg muscles (complete Conus and Cauda Equina syndrome) the progression of muscle atrophy to degeneration and the extent of muscle recovery after experimental

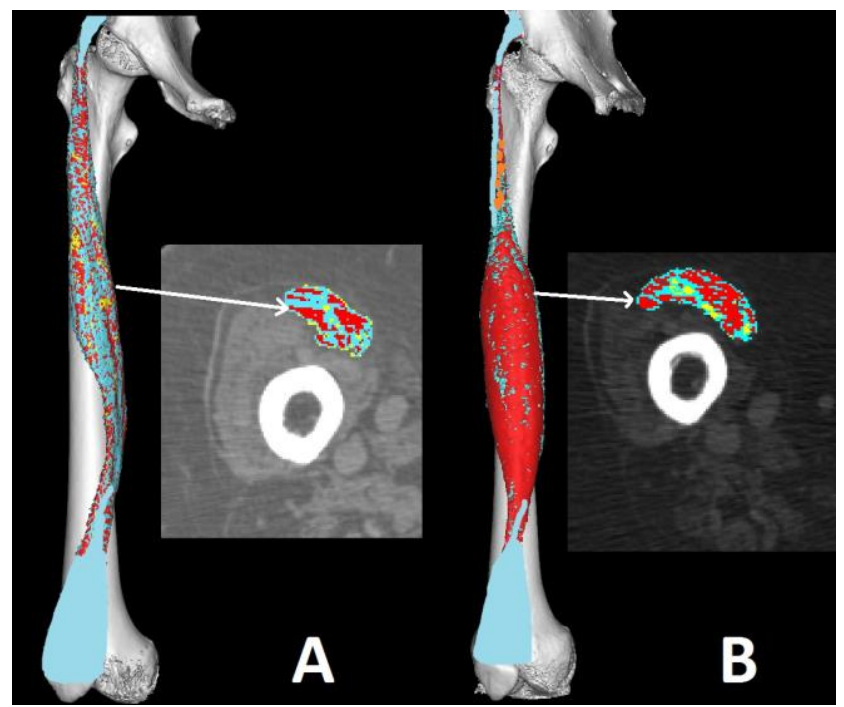

Fig 6. $2 D$ and $3 D$ false color muscle $C T$. Reconstructions at the cortical epimysial level of the Rectus femoris in false colors demonstrate the severe atrophy of a four years denervated muscle in case of complete Conus and Cauda Equina syndrome due to trauma to the spinal cord, and the extent of recovery after additional four years of home-based Functional Electrical Stimulation ( $h-b$ FES). The cross sections confirm that the whole muscle recovered from atrophy. ${ }^{25-32}$

therapy. ${ }^{29-32}$ Changes in tissue composition within the muscle were visualized by associating different colors to Hounsfield unit values of normal or atrophic muscle, fat and connective loose and fibrous tissue. The results of this analysis were presented as the percentage of different tissues (muscle, loose and fibrous connective tissue, and fat) in cross-sections and in the total volume of muscle on the three-dimensional reconstructions. Authors stress that the advantages of these approaches outweigh the low risk related to irradiation, in particular during follow-up of supervised trials, adding quantitative evidence to clinical assessments.

As mentioned before, in clinical practice it is possible to use rating scales in order to semi-quantitatively assess the degree of muscle degeneration. This type of scaling evaluates the extension of fatty degeneration. Its use is relatively quick and has a good inter-observer and intra-observer agreements. ${ }^{2,33}$

One of the most used is a 5-point scale where the signal intensity, evaluated in MRI T1-weighted sequence, is scored using a modification of the scale reported by Lamminen (Table 1 and Figure 7) ) $^{6,34}$ In the early stages of disease or in mild cases, an MR scan may be normal or slightly pathological, with a selective involvement of a few muscles. In more advanced myopathies, the pattern of involvement is less evident 
Clinical imaging in genetic neuromuscular disorders

Eur J Transl Myol - Basic Appl Myol 2015; 25 (2): 121-127

Table 1. Modified Lamminen rating scale.

\begin{tabular}{|c|c|}
\hline SCORE & DESCRIPTION \\
\hline 0 & Normal \\
\hline 1 & Mild with only traces of increased signal intensity \\
\hline 2 & Moderate with increased signal in less than $50 \%$ of affected muscle \\
\hline 3 & Severe with increased signal intensity in more than $50 \%$ of affected muscle \\
\hline 4 & Entire muscle replaced by abnormal signal \\
\hline
\end{tabular}

because more muscles are involved; but, spared muscles or less affected ones, which identify the socalled "negative pattern", can be useful diagnostic clues. ${ }^{11}$ For diagnostic purposes, a quantitative description of the muscle involvement is not necessary, as the degree of fatty degeneration correlates with the severity and duration of the disease. The articles describing the pattern of involvement with the MRI, in different types of myopathy and dystrophy, have a low number of patients so far, and little is known about the spectrum of disease, which is only detectable by studies with higher number of patients. Furthermore, when MRI indications of the disease can be identified, the degree of disease progression assessed by MRI, whether sex, ethnicity, exercising can influence the progression and whther the pattern varies with the type of mutation in a specific gene are not fully understood. Knowing these points would help with diagnoses and would give useful information about the pathophysiology and therapy of neuromuscular diseases. ${ }^{22}$ MRI is increasingly used in the evaluation of patients with genetic neuromuscular diseases. Thanks to its high sensitivity in the detection of muscular abnormalities and a good inter-observer and intra-observer agreements, ${ }^{2} \mathrm{MRI}$ is preferred to CT and
US. $^{6}$ In these cases, semi-quantitative rating scales allowfor the objective assessment of muscle degeneration and therefore MRI provides an alternative or supportive analysis in follow up aside from clinical and laboratory tests. In patients suspected to suffer or suffering genetic neuromuscular disorders, MRI is, thus, useful for: 1. identifying asymptomatic patients, but with RM signs of muscle involvement (e.g. family members of asymptomatic patients or patients susceptible to malignant hyperthermia); 2. Staging the extent of muscle damage; 3. guiding muscle biopsy, which allows for the selection of the more diseased muscle or muscle group; 4. selecting therapeutic treatments and evaluating new therapies; 5. monitoring disease progression and following-up with patients.

In conclusion, radiologic imaging is increasingly playing a relevant role in neuromuscular disorders: in particular, those of known or unknown genetic etiology. In spite of the dominant role of Magnetic Resonance Imaging, other approaches maintain important roles: in particular, Ultrasoun with reagards to in guiding muscle biopsy. Other promising applications are described in details in other chapters of this Ejtm Special: News of Clinical Muscle Imaging.

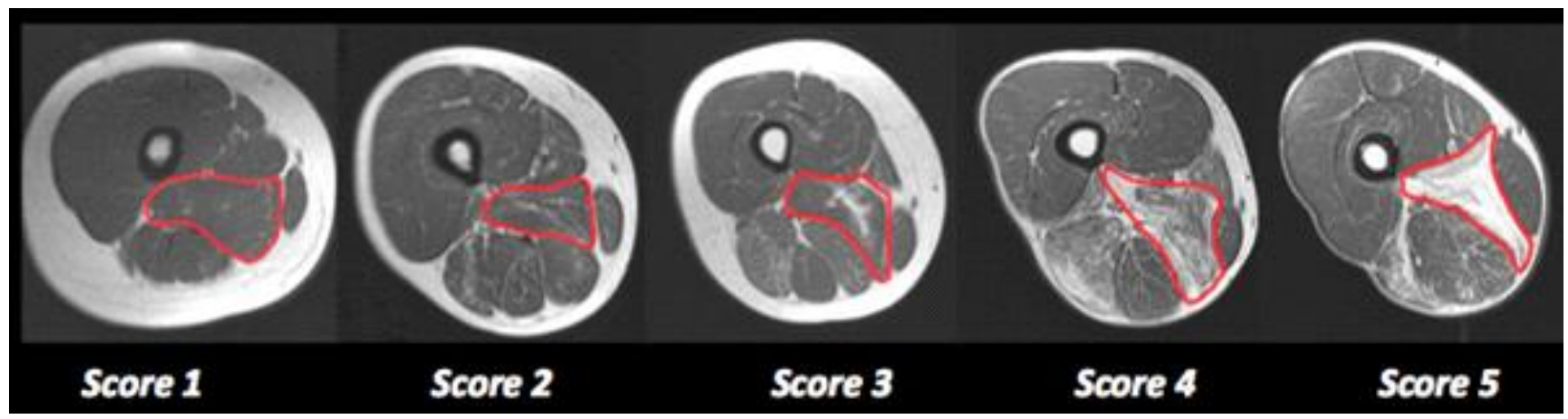

Fig 7. Modified Lamminen grading scale. Axial T1-weighted images of the thigh showing different grade of fatty infiltration according to the grading scale. 


\section{Clinical imaging in genetic neuromuscular disorders}

Eur J Transl Myol - Basic Appl Myol 2015; 25 (2): 121-127

\section{Acknowledgement}

Authors are indebted with Paolo Gargiulo, Rejkyavik (Iceland) for permission to reproduce figure 6 . The authors gratefully thank Kyle J. Edmunds for his critical reading of the typescript.

\section{Corresponding Author}

Paolo Ortolan, Radiology Unit, Department of Medicine, University of Padova, Italy

Email: paolo.ortolan@gmail.com

E-mails of Co-Authors:

Riccardo Zanato, riccardo_zanato@yahoo.it

Alessandro Coran: alecoran@gmail.com

Valeria Beltrame: valeria.beltrame@unipd.it

Roberto Stramare, roberto.stramare@unipd.it

\section{References}

1. Quijano-Roy S, Avila-Smirnow D, Carlier RY WB-MRI muscle study group. Whole body muscle MRI protocol: Pattern recognition in early onset NM disorders. Neuromuscul Disord 2012;22 Suppl 2:S68-84.

2. Wattjes MP, Kley RA, Fischer D. Neuromuscular imaging in inherited muscle diseases. Eur Radiol 2010;20:2447-60.

3. Schedel $H$, Reimers $C D$, Nagele $M$, et al. Imaging techniques in myotonic dystrophy. A comparative study of ultrasound, computed tomography and magnetic resonance imaging of skeletal muscles. Eur J Radiol 1992;15:230-8.

4. Termote JL, Baert A, Crolla D, et al. Computed tomography of the normal and pathologic muscular system. Radiology 1980;137:439-44.

5. Messineo D, Cremona A, Trinci M, et al. MRI in the study of distal primary myopathopies and of muscular alterations due to peripheral neuropathies: Possible diagnostic capacities of MR equipment with low intensity field $(0.2 \mathrm{~T})$ dedicated to peripheral limbs. Magn Reson Imaging 1998;16:731-41.

6. Lamminen AE. Magnetic resonance imaging of primary skeletal muscle diseases: Patterns of distribution and severity of involvement. $\mathrm{Br} \mathrm{J}$ Radiol 1990;63(756):946-50.

7. Castillo J, Pumar JM, Rodriguez JR, et al. Magnetic resonance imaging of muscles in myotonic dystrophy. Eur J Radiol 1993;17:1414.

8. Mercuri E, Pichiecchio A, Allsop J, et al. Muscle MRI in inherited neuromuscular disorders: Past, present, and future. J Magn Reson Imaging 2007;25:433-40.

9. Alanen AM, Falck B, Kalimo H, et al. Ultrasound, computed tomography and magnetic resonance imaging in myopathies: Correlations with electromyography and histopathology.

Acta

Neurol

Scand 1994;89:336-46

10. Schedel H, Reimers $C D$, Nägele $M$, et al. Imaging techniques in myotonic dystrophy. A comparative study of ultrasound, computed tomography and magnetic resonance imaging of skeletal muscles. Eur J Radiol. 1992;15:230-8.

11. Pillen S, Arts IM, Zwarts MJ. Muscle ultrasound in neuromuscular disorders. Muscle Nerve. 2008;37:679-93.

12. Pillen S. Skeletal muscle ultrasound. Basic Appl Myol - Eur J Transl Myol 2010; 20: 145-156.

13. Maggi L, Scoto M, Cirak S, et al. Congenital myopathies--clinical features and frequency of individual subtypes diagnosed over a 5-year period in the united kingdom. Neuromuscul Disord 2013;23:195-205.

14. Heckmatt JZ, Dubowitz V. Ultrasound imaging and directed needle biopsy in the diagnosis of selective involvement in muscle disease. J Child Neurol 1987;2:205-13.

15. O'Sullivan PJ, Gorman GM, Hardiman OM, et al. Sonographically guided percutaneous muscle biopsy in diagnosis of neuromuscular disease: A useful alternative to open surgical biopsy. J Ultrasound Med 2006;25:1-6.

16. Pillen S, van Keimpema M, Nievelstein RA, et al. Skeletal muscle ultrasonography: Visual versus quantitative evaluation. Ultrasound Med Biol 2006;32:1315-21.

17. Quijano-Roy S, Carlier RY, Fischer D. Muscle imaging in congenital myopathies. Semin Pediatr Neurol 2011;18:221-9.

18. Cupler EJ, Bohlega S, Hessler R, et al. Miyoshi myopathy in saudi arabia: Clinical, electrophysiological, histopathological and radiological features. Neuromuscul Disord 1998;8:321-6.

19. Lamminen AE, Tanttu JI, Sepponen RE, et al. Magnetic resonance of diseased skeletal muscle: Combined T1 measurement and chemical shift imaging. Br J Radiol 1990;63:591-6.

20. Straub V, Donahue KM, Allamand V, et al. Contrast agent-enhanced magnetic resonance imaging of skeletal muscle damage in animal models of muscular dystrophy. Magn Reson Med 2000;44:655-9.

21. Stramare R, Beltrame V, Dal Borgo R, et al: MRI in the assessment of muscular pathology: a comparison between limb-girdle muscular dystrophies, hyaline body myopathies and myotonic dystrophies. Radiol Med 2010;115:585-99. doi: 10.1007/s11547-0100531-2. Epub 2010 Feb 22. English, Italian.

22. Straub V, Carlier PG, Mercuri E. TREAT-NMD workshop: Pattern recognition in genetic muscle diseases using muscle MRI: 2011, Rome, Italy. Neuromuscul Disord 2012;22 Suppl 2:S42-53. 


\section{Clinical imaging in genetic neuromuscular disorders}

Eur J Transl Myol - Basic Appl Myol 2015; 25 (2): 121-127

23. Beltrame V, Ortolan P, Coran A, Zanato R, Gazzola M, Frigo A, Bello L, Pegoraro E, Stramare R. Muscle MR imaging in tubular aggregate myopathy: PLoS One. 2014 Apr 10;9(4):e94427. doi: 10.1371/journal.pone. 0094427. eCollection 2014.

24. Klein A, Jungbluth H, Clement E, etal.. Muscle magnetic resonance imaging in congenital myopathies due to ryanodine receptor type 1 gene mutations. Arch Neurol 2011;68:1171-9. doi:10.1001/archneurol.2011.188.)

25. Gargiulo $\mathrm{P}$, Helgasson $\mathrm{B}$, Magnússon $\mathrm{B}$, et al. Monitoring Bone Density from Patients Undergoing Total Hip Arthroplasty using Computed Tomography and 3D Modeling Techniques. Biomed Tech (Berl). 2013 Sep 7. pii: /j/bmte.2013.58.issue-s1-S/bmt-20134419/bmt-2013-4419.xml. doi: 10.1515/bmt2013-4419.

26. Gargiulo P, Reynisson PJ, Helgason B, et al. Muscle, tendons, and bone: structural changes during denervation and FES treatment. Neurol Res 2011;33:750-8. doi: 10.1179/1743132 811Y.0000000007.

27. Gargiulo P, Helgason $\mathrm{T}$, Reynisson PJ, et al. Monitoring of muscle and bone recovery in spinal cord injury patients treated with electrical stimulation using three-dimensional imaging and segmentation techniques: methodological assessment. Artif Organs 2011;35:275-81. doi: 10.1111/j.1525-1594.2011.01214.x.

28. Gargiulo P, Kern H, Carraro U, et al. Quantitative color three-dimensional computer tomography imaging of human long-term denervated muscle. Neurol Res 2010;32:13-9. doi: 10.1179/016164109 X12536042424171.

29. Kern H, Carraro U, Adami N, et al. Homebased Functional Electrical Stimulation (hbFES) recovers permanently denervated muscles in paraplegic patients with complete lower motor neuron lesion. Neurorehab Neur Rep 2010;24:709-21.

30. Gargiulo P, Vatnsdal B, Ingvarsson $\mathrm{P}$, et al. Restoration of muscle volume and shape induced by electrical stimulation of denervated degenerated muscles: qualitative and quantitative measurement of changes in rectus femoris using computer tomography and image segmentation. Artif Organs 2008;32:609-13.

31. Gargiulo P, Vatnsdal B, Ingvarsson $P$, et al. Computational methods to analyse tissue composition and structural changes in denervated muscle undergoing therapeutic electrical stimulation. Basic Appl Myol/ European Journal of Translational Myology 2009;19:157-62.

32. Gargiulo P, Kern H, Carraro U, et al. Quantitative colour 3D CT imaging of human long-term denervated muscle. Progression to fibrosis of perimysium and a case report of FES recovery. Neurol Res 2010;32:13-9.

33. Mercuri E, Jungbluth H, Muntoni F. Muscle imaging in clinical practice: Diagnostic value of muscle magnetic resonance imaging in inherited neuromuscular disorders. Curr Opin Neurol 2005;18:526-37.

34. Lamminen AE. Magnetic resonance imaging of primary skeletal muscle diseases: patterns of distribution and severity of involvement $\mathrm{Br} \mathrm{J}$ Radiol 1990;63:946-50.

35. Edmunds KJ, Gargiulo P. Imaging approaches in noninvasive functional assessment of implantable myogenic biomaterials and engineered muscle tissue. Eur J Transl Myol Basic Appl Myol 2015;25:63-76.

36. Wiedemann L, Chaberova J, Edmunds KJ, et al. Low-amplitude craniofacial EMG power spectral density and 3D muscle reconstruction from MRI. Eur J Transl Myol - Basic Appl Myol 2015;25:93-9.

37. Magnússon B, Pétursson P, Kyle Edmunds KJ, et al. Improving planning and post-operative assessment for Total Hip Arthroplasty. Eur J Transl Myol - Basic Appl Myol 2015;25:101-8

38. Carraro U, Edmunds KJ, Gargiulo P. 3D False Color Computed Tomography for diagnosis and follow-up of permanent denervated human muscles submitted to Functional Electrical Stimulation. Eur J Transl Myol - Basic Appl Myol 2015;25:129-140. 